\title{
Extending Offset-Based Response-Time Analysis for Mixed Messages in Controller Area Network
}

\author{
Saad Mubeen*†, Jukka Mäki-Turja*† and Mikael Sjödin* \\ * Mälardalen Real-Time Research Centre (MRTC), Mälardalen University, Västerås, Sweden \\ ${ }^{\dagger}$ Arcticus Systems AB, Järfälla, Sweden \\ \{saad.mubeen, jukka.maki-turja, mikael.sjodin\}@mdh.se
}

\begin{abstract}
The existing offset-based response-time analysis for mixed messages in Controller Area Network (CAN) assumes the jitter and deadline of a message to be smaller or equal to the transmission period. However, practical systems may contain messages whose release jitter and deadlines can be greater than their periods, e.g., in the gateway nodes. We extend the existing response-time analysis for mixed messages in CAN that are scheduled with offsets and have arbitrary jitter and deadlines. Mixed messages are implemented by several higher-level protocols for CAN that are used in the automotive industry. The extended analysis is applicable to any higher-level protocol for CAN that uses periodic, sporadic and mixed transmission modes.
\end{abstract}

\section{Introduction}

The Controller Area Network (CAN) [26] is a multimaster, event-triggered, serial communication bus protocol supporting bus speeds of up to 1 mega bits per second. It is a widely used protocol in the automotive domain. It has been standardized as ISO 11898-1 [18]. According to CAN in Automation (CiA) [1], the estimated number of CAN enabled controllers sold in 2011 are about 850 million. CAN also finds its applications in other domains, e.g., industrial control, medical equipments, maritime electronics, and production machinery. There are several higher-level protocols for CAN that are developed for many industrial applications such as CAN Application Layer (CAL), CANopen, J1939, Hägglunds Controller Area Network (HCAN), CAN for Military Land Systems domain (MilCAN).

In order to provide evidence that each action by the system will be provided in a timely manner, i.e., each action will be taken at a time that is appropriate to the environment of the system, a priori analysis techniques such as schedulability analysis have been developed by the research community. Response-Time Analysis (RTA) [19, 9, 8, 28] is a powerful, mature and well established schedulability analysis technique. It is a method to calculate upper bounds on the response times of tasks or messages in a real-time system or a network respectively. RTA is used to perform a schedulability test which means it checks whether or not tasks (or messages) in the system (or network) will satisfy their deadlines. RTA applies to systems (or networks) where tasks (or messages) are scheduled with respect to their priorities and which is the predominant scheduling technique used in real-time operating systems (or real-time network protocols, e.g., CAN) [24].

\subsection{Motivation and related work}

Tindell et al. [31] developed the schedulability analysis for CAN. This analysis has been implemented in several tools that are used in the automotive industry [6, 21, 23, 5]. Davis et al. [12] refuted, revisited and revised the analysis by Tindell et al. In [13], Davis et al. extended the analysis in $[31,12]$ which is applicable to the CAN network where some nodes implement priority queues and some implement FIFO queues. All these analyses assume that the messages are queued for transmission periodically or sporadically. They do not support mixed messages in CAN, i.e., the messages that are simultaneously time (periodic) and event (sporadic) triggered. Mubeen et al. [20] extended the existing analysis to support mixed messages in CAN where nodes implement priority-based queues. Mubeen et al. [27] further extended their analysis to support mixed messages in the network where some nodes implement priority queues while others implement FIFO queues.

But, none of the analysis discussed above supports messages that are scheduled with offsets, i.e., using externally imposed delays between the times when the messages can be queued. In order to avoid deadlines violations due to high transient loads, current automotive embedded systems are often scheduled with offsets [32]. Furthermore, the worst-case response times of messages (especially with lower priority) in CAN increase with the increase in the network load. However, the worst-case response-times of lower priority messages in CAN can be reduced if the messages are scheduled with offsets $[29,15,16]$. A method for the assignment of offsets to improve the overall bandwidth utilization is proposed in $[15,16]$. The worst-case response-time analysis for CAN messages with offsets has been developed by several researchers [11, 14, 29, 17, 32].

None of these analyses support mixed messages that are scheduled with offsets. In [22], we extended the existing 


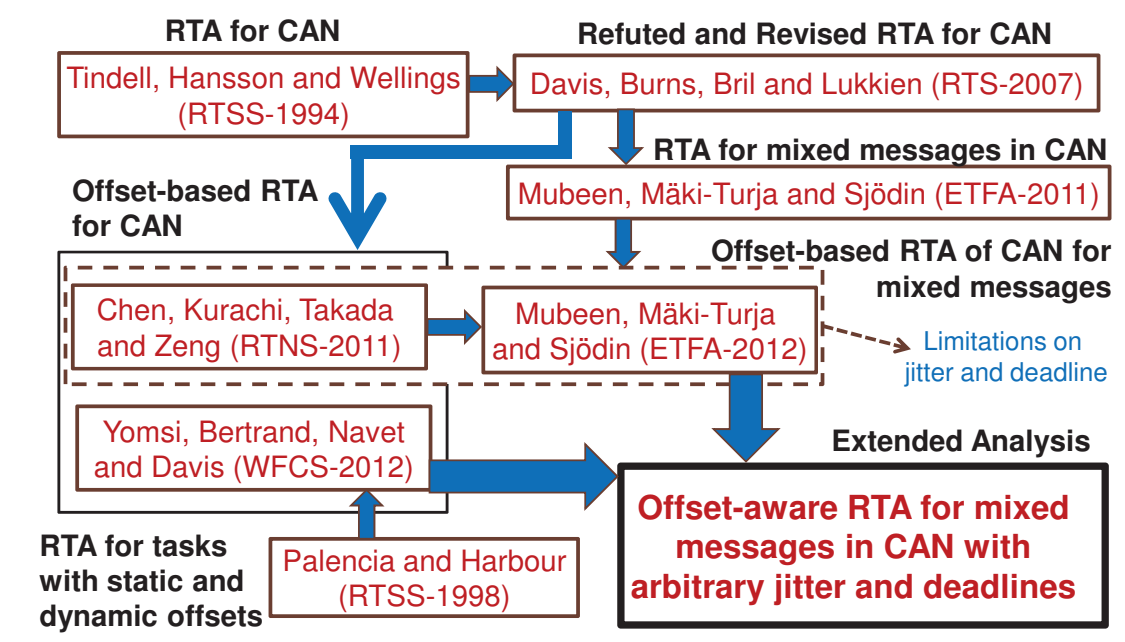

Figure 1. Relation between the existing and extended Response Time Analysis (RTA)

offset-based analysis [11] to support worst-case responsetime calculations for mixed messages in CAN. However, this analysis is restricted due to limitations regarding message jitter and deadlines. The source of these limitations comes from the base analysis [11]. In this paper, we remove these limitations. Basically, we extend the analysis for mixed messages [20] by building it upon the analysis for CAN messages with offsets [32]. Figure 1 depicts the relation between the existing and extended analyses.

\subsection{Paper contribution}

We extend the response-time analysis of CAN for mixed messages that are scheduled with offsets. The existing analysis for mixed messages with offsets [22] places restrictions on message deadline and jitter, i.e., each of them should be less than or equal to message period. Release jitter of a message can be higher than its transmission period in practical systems. For example, consider a message is exchanged between two networks (or two segments of a network) via a gateway node. At the gateway node, the message inherits its response time on the incoming network as part of its release jitter on the outgoing network. This release jitter can be higher than the period of the message [32]. The existing offset-based analysis does not support mixed messages whose jitter and deadlines are higher than their transmission periods.

In this paper, we lift these restrictions by assuming deadline and jitter to be arbitrary, i.e., each one of them can be higher than message period. Intuitively, there can be several instances of the same message that are queued for transmission. Hence, our extended analysis considers the response times of all these instances while calculating the worst-case response time. Mixed messages are implemented by several higher-level protocols used in the industry. The analysis is applicable to any higher-level protocol for CAN that uses periodic, sporadic and mixed transmission of messages that are scheduled with offsets. We also show the applicability of the extended analysis by conducting the automotive-application case study.

\subsection{Paper layout}

The remainder of the paper is organized as follows. In Section 2, we discuss mixed transmission patterns supported by higher-level protocols for CAN. Section 3 describes the scheduling model. Section 4 presents the extended analysis. In Section 5, we present a case study. Section 6 concludes the paper and discusses the future work.

\section{Mixed transmission patterns supported by higher-level protocols}

When CAN is employed for network communication in a distributed real-time system, each node (processor) or Electronic Control Unit (ECU) is equipped with a CAN interface that connects the node to the bus [30]. Application tasks in each node, that require remote transmission, are assumed to queue messages for transmission over the CAN network. The messages are transmitted according to the protocol specification of the CAN protocol. Traditionally, it is assumed that the tasks queueing CAN messages are invoked either by periodic events with a period or sporadic events with a minimum inter-arrival time. However, there are some higher-level protocols and commercial extensions of CAN in which the task that queues the messages can be invoked periodically as well as sporadically. If a message can be queued for transmission periodically as well as at the arrival of a sporadic event then the transmission type of the message is said to be mixed. In other words, a mixed message is simultaneously time (periodic) and event triggered (sporadic). We identified three types of implementations of mixed messages used in the industry.

Consistent terminology. To stay consistent, we use the terms message and frame interchangeably because we only consider messages that will fit into one frame (maximum 8 bytes). For the purpose of using simple notation, we call a CAN message as periodic, sporadic or mixed if it is queued by an application task that is invoked periodically, sporadically or both (periodically and sporadically) respectively. If a message is queued for transmission at periodic intervals, we use the term "Period" to refer to its periodic- 


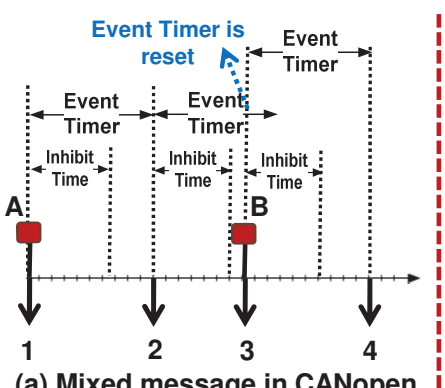

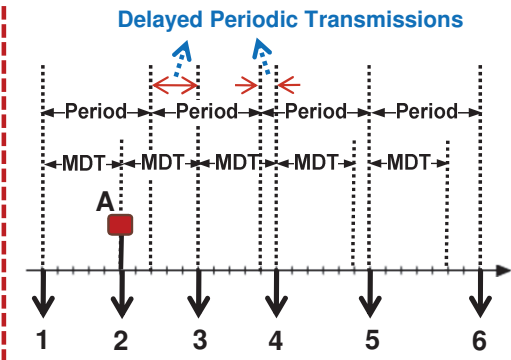

(b) Mixed message in AUTOSAR

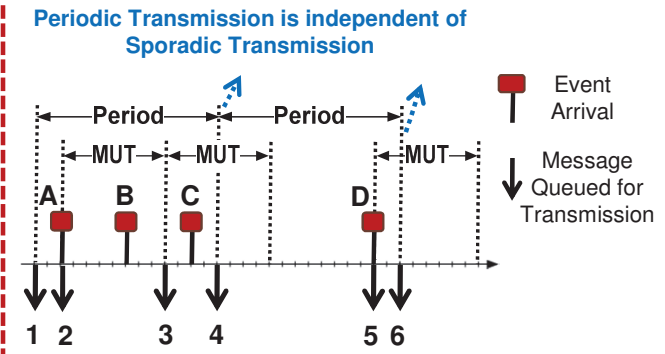

(c) Mixed message in HCAN

Figure 2. Mixed transmission pattern in higher-level protocols for CAN

ity. A sporadic message is queued for transmission as soon as an event occurs that changes the value of one or more signals contained in the message provided the Minimum Update Time (MUT) between queueing of two successive sporadic messages has elapsed. Hence, the transmission of a sporadic frame is constrained by the $M U T$. We overload the term "MUT" to refer to the "Inhibit Time" in CANopen protocol [3] and the "Minimum Delay Time (MDT)" in AUTOSAR communication [4].

\subsection{Method 1: Implementation in CANopen}

The CANopen protocol [3] supports mixed transmission that corresponds to the Asynchronous Transmission Mode coupled with the Event Timer. The Event Timer is used to transmit an asynchronous message cyclically. A mixed message can be queued for transmission at the arrival of an event provided the Inhibit Time has expired. The Inhibit Time is the minimum time that must be allowed to elapse between the queueing of two consecutive messages. A mixed message can also be queued periodically at the expiry of the Event Timer. The Event Timer is reset every time the message is queued. Once a mixed message is queued, any additional queueing of it will not take place during the Inhibit Time [3].

The transmission pattern of a mixed message in CANopen is illustrated in Figure 2(a). The down-pointing arrows symbolize the queueing of messages while the upward lines (labeled with alphabetic characters) represent arrival of the events. Message 1 is queued as soon as the event $A$ arrives. Both the Event Timer and Inhibit Time are reset. As soon as the Event Timer expires, message 2 is queued due to periodicity and both the Event Timer and Inhibit Time are reset again. When the event $B$ arrives, message 3 is immediately queued because the Inhibit Time has already expired. Note that the Event Timer is also reset at the same time when message 3 is queued as shown in Figure 2(a). Message 4 is queued because of the expiry of the Event Timer. There exists a dependency relationship between the Inhibit Time and the Event Timer, i.e., the Event Timer is reset not only with every periodic transmission but also with every sporadic transmission.

\subsection{Method 2: Implementation in AUTOSAR}

AUTOSAR (AUTomotive Open System ARchitecture) [2] can be viewed as a higher-level protocol if it uses CAN for network communication. Mixed transmission mode in AUTOSAR is widely used in practice. In AUTOSAR, a mixed message can be queued for transmission repeatedly with a period equal to the mixed transmission mode time period. The mixed message can also be queued at the arrival of an event provided the Minimum Delay Time $(M D T)$ has been expired. However, each transmission of the mixed message, regardless of being periodic or sporadic, is limited by the $M D T$. This means that both periodic and sporadic transmissions are delayed until the $M D T$ expires. The transmission pattern of a mixed message implemented by AUTOSAR is illustrated in Figure 2(b). Message 1 is queued (the MDT is started) because of partly periodic nature of the mixed message. When the event $A$ arrives, message 2 is queued immediately because the $M D T$ has already expired. The next periodic transmission is scheduled 2 time units after the transmission of message 2. However, the next two periodic transmissions corresponding to messages 3 and 4 are delayed because the $M D T$ is not expired. This is indicated by the comment "Delayed Periodic Transmissions" in Figure 2(b). The periodic transmissions corresponding to messages 5 and 6 occur at the scheduled times because the $M D T$ is already expired in both cases.

\subsection{Method 3: Implementation in HCAN}

A mixed message in HCAN protocol [7] contains signals out of which some are periodic and some are sporadic. A mixed message is queued for transmission not only periodically but also as soon as an event occurs that changes the value of one or more event signals, provided the $M U T$ between the queueing of two successive sporadic instances of the mixed message has elapsed. Hence, the transmission of the mixed message due to arrival of events is constrained by the MUT. The transmission pattern of the mixed message is illustrated in Figure 2(c). Message 1 is queued because of periodicity. As soon as event $A$ arrives, message 2 is queued. When event $B$ arrives it is not queued immediately because the $M U T$ is not expired yet. As soon as the $M U T$ expires, message 3 is queued. Message 3 contains the signal changes that correspond to event $B$. Similarly, a message is not immediately queued when the event $C$ arrives because the $M U T$ is not expired. Message 4 is queued because of the periodicity. Although, the $M U T$ was not expired, the event signal corresponding to event $C$ 
was packed in message 4 and queued as part of the periodic message. Hence, there is no need to queue an additional sporadic message when the MUT expires. This indicates that the periodic transmission of the mixed message cannot be interfered by its sporadic transmission (a unique property of the HCAN protocol). When the event $D$ arrives, a sporadic instance of the mixed message is immediately queued as message 5 because the MUT has already expired. Message 6 is queued due to periodicity.

\subsection{Discussion}

In the first method, the Event Timer is reset every time the mixed message is queued for transmission. The implementation of mixed message in method 2 is similar to method 1 to some extent. The main difference is that in method 2, the periodic transmission can be delayed until the expiry of the $M D T$. Whereas in method 1 , the periodic transmission is not delayed, in fact, the Event Timer is restarted with every sporadic transmission. The $M D T$ timer is started with every periodic or sporadic transmission of the mixed message. Hence, the worst-case periodicity of the mixed message in methods 1 and 2 can never be higher than the Inhibit Timer and MDT respectively. This means that the models of mixed messages in the first and second implementation methods reduce to the classical sporadic model. Therefore, the existing analyses for CAN messages with offsets $[11,14,29,17,32]$ can be used for analyzing mixed messages in the first and second implementation methods.

However, the periodic transmission is independent of the sporadic transmission in the third method. The periodic timer is not reset with every sporadic transmission. The mixed message can be queued for transmission even if the $M U T$ is not expired. Hence, the worst-case periodicity of the mixed message is neither bounded by period nor by the $M U T$. Therefore, the analyses in $[11,14,29,17,32]$ cannot be used for analyzing the mixed messages in the third implementation method.

\section{System model}

The system, $S$, consists of a number of CAN controllers that are connected to a single CAN network. The nodes implement priority-ordered queues, i.e., the highest priority message in a node enters into the bus arbitration. Each CAN message $m_{m}$ has a unique identifier and a priority denoted by $I D_{m}$ and $P_{m}$ respectively. The priority of a message is assumed to be equal to its ID. The priority of $m_{m}$ is considered higher than the priority of $m_{n}$ if $P_{m}<P_{n}$.

Let the sets $h p\left(m_{m}\right), l p\left(m_{m}\right)$, and $h e p\left(m_{m}\right)$ contain the messages with priorities higher, lower, and equal and higher than $m_{m}$ respectively. Although the priorities of CAN messages are unique, the set $h e p\left(m_{m}\right)$ will be used in the case of mixed messages. Associated to each message is a FRAME_TYPE that specifies whether the frame is a standard or an extended CAN frame. The difference between the two frame types is that the standard CAN frame uses an 11-bit identifier whereas the extended CAN frame uses a 29-bit identifier. In order to keep the notations simple and consistent, we define a function $\xi_{m}$ that denotes the transmission type of a message. $\xi_{m}$ specifies whether $m$ is periodic $(P)$, sporadic $(S)$ or mixed $(M)$. Formally, the domain of $\xi_{m}$ can be defined as follows.

$$
\xi_{m} \in[P, \quad S, \quad M]
$$

Each message $m_{m}$ has a transmission time $C_{m}$ and queueing jitter $J_{m}$ which is inherited from the task that queues $m_{m}$, i.e., the sending task. We assume that $J_{m}$ can be smaller, equal or greater than $T_{m}$ or $M U T_{m}$. Each message can carry a data payload that ranges from 0 to 8 bytes. This number is specified in the header field of the frame called Data Length Code and is denoted by $s_{m}$. In the case of periodic transmission, $m_{m}$ has a transmission period which is denoted by $T_{m}$. Whereas in the case of sporadic transmission, $m_{m}$ has the $M U T_{m}$ (Minimum Update Time) that refers to the minimum time that should elapse between the transmission of any two sporadic messages. $B_{m}$ denotes the blocking time of $m_{m}$ which refers to the largest amount of time $m_{m}$ can be blocked by any lower priority message.

We duplicate a message when its transmission type is mixed. Hence, each mixed message $m_{m}$ is treated as two separate messages, i.e., one periodic and the other sporadic. The duplicates share all the attributes except the $T_{m}$ and $M U T_{m}$. The periodic copy inherits $T_{m}$ while the sporadic copy inherits the $M U T_{m}$. Each message has a worst-case response time, denoted by $R_{m}$, and defined as the longest time between the queueing of the message (on the sending node) and the delivery of the message to the destination buffer (on the destination node). $m_{m}$ is deemed schedulable if its $R_{m}$ is less than or equal to its deadline $D_{m}$. The system is considered schedulable if all of its messages are schedulable. We consider the deadlines to be arbitrary which means that they can be greater than the periods or MUTs of corresponding messages. We assume that the CAN controllers are capable of buffering more than one instance of a message. The instances of a message are assumed to be transmitted in the same order in which they are queued (i.e., we assume FIFO policy among the instances of the same message).

Let $O_{m}$ denotes the offset of $m_{m}$. We assume that the offset of $m_{m}$ is always smaller than its period. The first arrival time of $m_{m}$ is equal to its offset while the subsequent arrivals occur periodically with respect to the first arrival. We assume that the smallest offset in a node is zero. It should be noted that each node has its own local time and there is no global synchronization among the nodes. We assume that the offset relations exist only among periodic messages and periodic copies of mixed messages within a node. We further assume that there are no offset relations:

1. among sporadic messages,

2. between a periodic message and a sporadic message,

3 . between a periodic copy of a mixed message and a sporadic message,

4. between the duplicates of a mixed message, 
5. between any two messages belonging to different nodes.

All periodic messages and periodic copies of mixed messages in a node are gathered into a single transaction denoted by $\Gamma_{i}$. Each transaction $\Gamma_{i}$ belongs to $\Gamma$ which is the set of all transactions in the system. This transactional model is adapted from [25]. It should be noted that the offset relations exist only within a transaction, and there are no offset relations among transactions. In the context of a transaction, we denote a message $m_{j}$ belonging to transaction $i$ by $m_{i}^{j}$. Each transaction has a period denoted by $T_{\Gamma_{i}}$ and defined as the Least Common Multiple (LCM) of the periods of all messages present in the transaction. Each sporadic message or sporadic copy of a mixed message is modeled as a transaction of its own.

An example of two messages transmitted by a node is depicted in Figure 3. $m_{1}$ is a mixed message with high priority while $m_{2}$ is a periodic message with low priority. Transaction $\Gamma_{1}$ contains both $m_{2}$ and periodic copy of $m_{1}$. The period of $\Gamma_{1}$ denoted by $T_{\Gamma_{i}}$ is the LCM of $T_{1}$ and $T_{2}$. Transaction $\Gamma_{2}$ consists of only the sporadic copy of $m_{1}$.

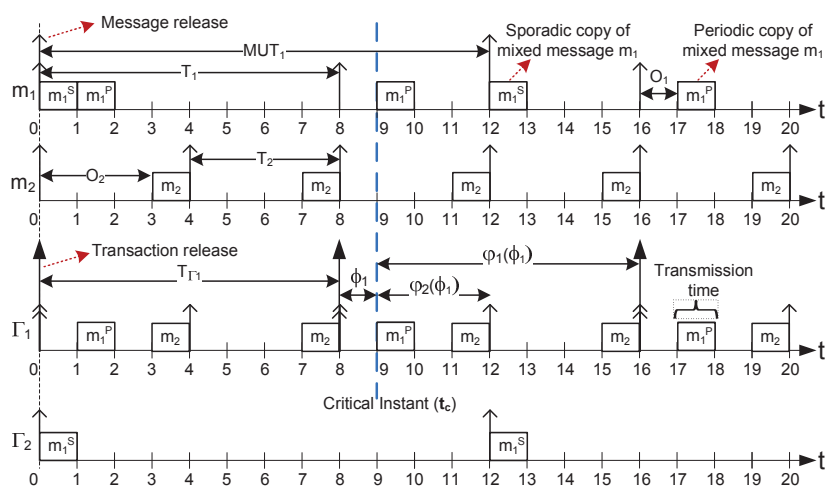

Figure 3. Example demonstrating messages and transactions

\section{Worst-case response-time analysis}

Let $m_{m}$ be the message under analysis. We analyze $m_{m}$ differently based on its transmission type. Intuitively, we consider three different cases namely periodic, sporadic and mixed. We discuss few terms that are used in the analysis. In order to calculate the worst-case response time of $m_{m}$, the maximum busy period $[31,12]$ for priority level-m should be known first.

Maximum Busy Period. It is the longest contiguous interval of time during which $m_{m}$ is unable to complete its transmission due to two reasons. First, the bus is occupied by the higher priority messages. Second, a lower priority message already started its transmission when $m_{m}$ is queued for transmission. The maximum busy period starts at the critical instant.

Critical Instant. For a system where messages are scheduled without offsets, the critical instant corresponds to the point in time when all higher priority messages are assumed to be queued simultaneously with $m_{m}$ while their subsequent instances are assumed to be queued after the shortest possible interval of time [12]. However, this assumption does not hold in the system where messages are scheduled with offsets.

We redefine the critical instant for priority level-m busy period as the instant when (1) $m_{m}$ or any other higher priority message belonging to the same node as that of $m_{m}$ is queued for transmission, (2) At least one message with priority higher than $m_{m}$ is queued for transmission from every node, (3) all sporadic messages and sporadic copies of mixed messages belonging to the set $h p\left(m_{m}\right)$ from every node are simultaneously queued for transmission at the respective nodes, and (4) a lower priority message just started its transmission when $m_{m}$ is queued. The critical instant for priority level-2 busy period is identified at $t_{c}$ in Figure 3. According to the condition (3) stated above, the arrival of $\Gamma_{2}$ should coincide with the critical instant.

Worst-Case Candidates. The main issue regarding the condition (2) is to determine which message in the set $h p\left(m_{m}\right)$ is the candidate to start the critical instant, i.e., contributing to the worst-case response-time of $m_{m}$. The solution is that any message in the set $h p\left(m_{m}\right)$ can be the worst-case candidate. Therefore, each message has to be tested in the busy period as the potential worst-case candidate. The response time of $m_{m}$ should be calculated from every worst-case candidate and the maximum among all should be considered as the worst-case response time of $m_{m}$.

We present the response-time analysis with respect to any worst-case candidate in the following subsections.

\subsection{Case: When $\boldsymbol{m}_{m}$ is a periodic message}

Let $m_{m}$ belongs to transaction $\Gamma_{i}$. The worst-case response time of $m_{m}$ is equal to the maximum value among the response times of all of its instances. We calculate the response times of all instances of $m_{m}$ within the priority level-m busy period. Let $q_{m}$ be the index variable to denote instances of $m_{m}$. Let $q_{m}^{L}$ and $q_{m}^{H}$ denote lowest- and highest-numbered instances respectively. The worst-case response time of $m_{m}$ is given by:

$$
R_{m}=\max \left\{R_{m}\left(q_{m}\right)\right\}, \quad \forall q_{m}^{L} \leq q_{m} \leq q_{m}^{H}
$$

It should be noted that $q_{m}$ will be equal to 1 if the message instance is queued for transmission between the critical instant and $T_{m}$. Further, $q_{m}$ will be equal to 2 if the message instance is queued for transmission between $T_{m}$ and 2. $T_{m}$. Similarly, $q_{m}$ will be equal to 0 if the message instance is queued for transmission between the critical instant and $-T_{m}$. Since the jitter of a message can be greater than its transmission period, it is possible that the previous instances of the message may also be delayed due to jitter and enter in the maximum busy period. The calculations for the response time of instance $q_{m}$ are adapted from [32] as follows.

$$
R_{m}\left(q_{m}\right)=S T_{m}+C_{m}-\left(\varphi_{m}\left(\phi_{i}\right)+\left(q_{m}-1\right) \cdot T_{m}\right)
$$

$\phi_{i}$ in (2) denotes the time interval between latest arrival 
of $\Gamma_{i}$ (prior to the critical instant) and the critical instant. Consider the example message set in Figure $3 . \phi_{i}$ is equal to 1 time unit and is identified as $\phi_{1}$ on the third time line from the top. $\varphi_{m}\left(\phi_{i}\right)$ in (2) represents the length of the time interval between the critical instant and first release of $m_{m}$ that occurs at or after the critical instant. Consider again the example message set in Figure 3. $\varphi_{m}\left(\phi_{i}\right)$ for messages $m_{1}^{P}$ and $m_{2}$ are identified by $\varphi_{1}\left(\phi_{1}\right)$ and $\varphi_{2}\left(\phi_{1}\right)$ respectively. The calculations for $\varphi_{m}\left(\phi_{i}\right)$ are adapted from [25] as follows.

$$
\varphi_{m}\left(\phi_{i}\right)=\left(T_{m}-\left(\phi_{i}-O_{m}\right) \quad \bmod T_{m}\right) \quad \bmod T_{m}
$$

$S T_{m}$ in (2) denotes the Start Time (ST) when the priority level-m busy period ends and $m_{m}\left(q_{m}\right)$ can start its transmission. Basically, it sums up the interferences due to higher priority messages, previous instances of the same message and the blocking factor. It can be calculated by solving the following equation.

$$
S T_{m}^{n+1}=B_{m}+\left(q_{m}-q_{m}^{L}\right) \cdot C_{m}+\sum_{\forall \Gamma_{k} \in \Gamma} W_{m}\left(\Gamma_{k}, \phi_{k}, S T_{m}^{n}\right)
$$

Where the term $\left(q_{m}-q_{m}^{L}\right) \cdot C_{m}$ represents the interference due to previous instances of $m_{m}$ that are queued ahead of the instance under analysis. $B_{m}$ is the blocking delay for $m_{m}$. It is defined as the amount of time equal to the largest transmission time in the set of lower priority messages. $B_{m}$ is calculated as follows.

$$
B_{m}=\max _{\forall m_{j} \in l p\left(m_{m}\right)}\left\{C_{j}\right\}
$$

(4) is an iterative equation. It is solved iteratively until two consecutive solutions become equal. The starting value for $S T_{m}^{n}$ in (4) can be selected equal to $B_{m}+\left(q_{m}-\right.$ $\left.q_{m}^{L}\right) . C_{m}$. In (4), $W_{m}$ represents the amount of interference due to the messages in the set $h p\left(m_{m}\right)$ that are queued for transmission since the beginning of the busy period. It is important to mention that a message cannot be interfered by higher priority messages during its transmission because CAN uses fixed-priority non-preemptive scheduling. Whenever we use the term interference, it refers to the amount of time $m_{m}$ has to wait in the send queue because the higher priority messages win the arbitration, i.e., the right to transmit before $m_{m}$. $W_{m}$ can be calculated as follows.

$$
W_{m}\left(\Gamma_{k}, \phi_{k}, S T_{m}^{n}\right)=\sum_{\forall m_{j} \in h p_{k}\left(m_{m}\right)} \Upsilon_{k}^{j}\left(S T_{m}^{n}\right) \cdot C_{j}
$$

Where $h p_{k}\left(m_{m}\right)$ represents the set of all those messages that belong to $\Gamma_{k}$ and have priority higher than $m_{m}$. $\Upsilon_{k}^{j}\left(S T_{m}^{n}\right)$ in (6) is calculated differently based on the transmission type $\xi_{j}$ of the higher priority message $m_{j}$. The calculations for $\Upsilon_{k}^{j}\left(S T_{m}^{n}\right)$ are adapted from [32] and [20] as follows. It should be noted that the existing analysis considers only higher priority periodic messages while calculating $\Upsilon_{k}^{j}\left(S T_{m}^{n}\right)$. On the other hand, we consider all higher priority periodic, sporadic and mixed messages while calculating $\Upsilon_{k}^{j}\left(S T_{m}^{n}\right)$.

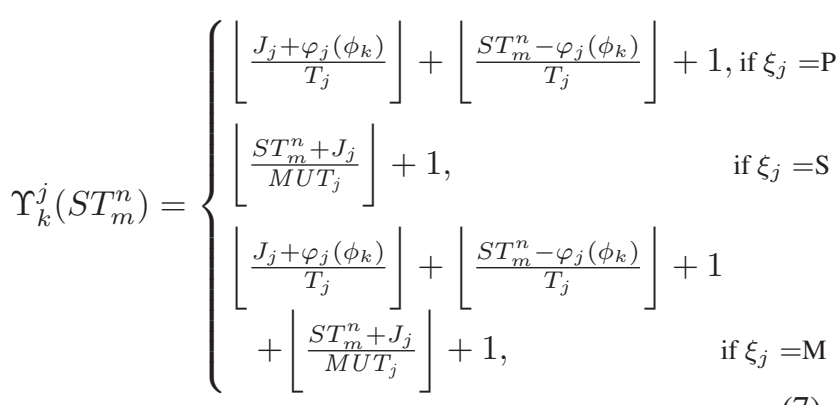

Where $\varphi_{j}\left(\phi_{k}\right)$ is calculated by replacing the indices $m$ and $i_{i}$ with ${ }_{j}$ and ${ }_{k}$ in (3) respectively. In (7), the periodic case is adapted from [32]. $\left\lfloor\frac{J_{j}+\varphi_{j}\left(\phi_{k}\right)}{T_{j}}\right\rfloor$ represents the maximum number of instances of the higher priority periodic message or periodic copy of mixed message $m_{j}$ that may accumulate at the critical instant. Whereas $\left\lfloor\frac{S T_{m}^{n}-\varphi_{j}\left(\phi_{k}\right)}{T_{j}}\right\rfloor+1$ represents the maximum number of instances of $m_{j}$ that are queued for transmission in the interval that starts with the critical instance and ends at $\Upsilon_{m}^{n}$.

There are no offset relations of $m_{m}$ with any sporadic message. Moreover, all sporadic messages are assumed to be queued for transmission at the critical instant. Therefore, the sporadic and mixed cases in (7) are adapted from [20]. $\left\lfloor\frac{S T_{m}^{n}+J_{j}}{M U T_{j}}\right\rfloor+1$ represent the maximum number of instances of higher priority sporadic message or sporadic copy of mixed message $m_{j}$ that are queued for transmission in the interval that starts with the critical instance and ends at $\Upsilon_{m}^{n}$. This also includes the number of instances of $m_{j}$ that may accumulate at the critical instant due to jitter.

It is evident from (7) that interference from both periodic and sporadic copies of every higher priority mixed message is taken into account. The lowest- and highestnumbered instances of $m_{m}$ denoted by $q_{m}^{L}$ and $q_{m}^{H}$ are calculated as follows.

$$
\begin{gathered}
q_{m}^{L}=-\left\lfloor\frac{J_{m}+\varphi_{m}\left(\phi_{i}\right)}{T_{m}}\right\rfloor+1 \\
q_{m}^{H}=\left\lceil\frac{L_{m}-\varphi_{m}\left(\phi_{i}\right)}{T_{m}}\right\rceil
\end{gathered}
$$

Where $L_{m}$ represents the length of priority level-m busy period. The existing analysis [32] considers only higher priority periodic messages while calculating $L_{m}$. We adapt the existing analysis to consider all higher priority periodic, sporadic and mixed messages while calculating $L_{m}$. Similar to (4), $L_{m}$ can be calculated using the fixed-point method as follows.

$$
\begin{aligned}
L_{m}^{n+1}= & {\left[\left\lfloor\frac{J_{m}+\varphi_{m}\left(\phi_{i}\right)}{T_{m}}\right\rfloor+\left\lceil\frac{L_{m}^{n}-\varphi_{m}\left(\phi_{i}\right)}{T_{m}}\right\rceil\right] \cdot C_{m}+} \\
& B_{m}+\sum_{\forall \Gamma_{k} \in \Gamma, m_{j} \in h p_{k}\left(m_{m}\right)} \mathrm{M}_{k}^{j}\left(L_{m}^{n}\right) \cdot C_{j}
\end{aligned}
$$


Where

$$
\mathrm{M}_{k}^{j}\left(L_{m}^{n}\right)= \begin{cases}\left\lfloor\frac{J_{j}+\varphi_{j}\left(\phi_{k}\right)}{T_{j}}\right\rfloor+\left\lceil\frac{L_{m}^{n}-\varphi_{j}\left(\phi_{k}\right)}{T_{j}}\right\rceil, & \text { if } \xi_{j}=\mathrm{P} \\ \left\lfloor\frac{L_{m}^{n}+J_{j}}{M U T_{j}}\right\rfloor+1, & \text { if } \xi_{j}=\mathrm{S} \\ \left\lfloor\frac{J_{j}+\varphi_{j}\left(\phi_{k}\right)}{T_{j}}\right\rfloor+\left\lceil\frac{L_{m}^{n}-\varphi_{j}\left(\phi_{k}\right)}{T_{j}}\right\rceil & \\ +\left\lfloor\frac{L_{m}^{n}+J_{j}}{M U T_{j}}\right\rfloor+1, & \text { if } \xi_{j}=\mathrm{M}\end{cases}
$$

\subsection{Case: When $\boldsymbol{m}_{m}$ is a sporadic message}

Let again the message under analysis be $m_{m}$ that belongs to $\Gamma_{i}$, i.e, the transaction of its own. The worst-case response time of $m_{m}$ can be calculated similar to the periodic case with one exception. That is, sporadic message does not hold any offset relations with any other message in the system. Moreover, all sporadic messages including $m_{m}$ are assumed to be queued for transmission at the critical instant. Intuitively, $\phi_{i}$ will be equal to $M U T_{m}$, i.e., the latest arrival of $m_{m}$ prior to critical instant will be $M U T_{m}$ time units before the critical instant. Let us use $O_{m}$ equal to zero, and $M U T_{m}$ in place of both $T_{m}$ and $\phi_{i}$ in (3).

$$
\varphi_{m}\left(\phi_{i}\right)=0
$$

In this case, (1), (4), (5), (6), (7) and (11) hold intact. However, we need to replace the new value of $\varphi_{m}\left(\phi_{i}\right)$ from (12) in the calculations for (2), (8), (9) and (10) as follows.

$$
\begin{gathered}
R_{m}\left(q_{m}\right)=S T_{m}+C_{m}-\left(q_{m}-1\right) \cdot M U T_{m} \\
q_{m}^{L}=-\left\lfloor\frac{J_{m}}{M U T_{m}}\right\rfloor+1 \\
q_{m}^{H}=\left\lceil\frac{L_{m}}{M U T_{m}}\right\rceil \\
L_{m}^{n+1}=\left\lfloor\left\lfloor\frac{J_{m}}{M U T_{m}}\right\rfloor+\left\lceil\frac{L_{m}^{n}}{M U T_{m}}\right\rceil\right] \cdot C_{m}+B_{m}+ \\
\forall \Gamma_{k} \in \Gamma, m_{j} \in h p_{k}\left(m_{m}\right)
\end{gathered}
$$

\subsection{Case: When $\boldsymbol{m}_{m}$ is a mixed message}

Let again $m_{m}$ be the message under analysis. Since a mixed message is duplicated as two separate messages, the extended analysis treats them separately. Let the periodic and sporadic copies of $m_{m}$ be denoted by $m_{m_{P}}$ and $m_{m_{S}}$ respectively. We denote the worst-case response times of $m_{m_{P}}$ and $m_{m_{S}}$ by $R_{m_{P}}$ and $R_{m_{S}}$ respectively. The worstcase response time of $m_{m}$ is the maximum between $R_{m_{P}}$ and $R_{m_{S}}$ as follows.

$$
R_{m}=\max \left\{R_{m_{P}}, R_{m_{S}}\right\}
$$

Where $R_{m_{P}}$ and $R_{m_{S}}$ are equal to the maximum value among the response times of their respective instances. Let
$q_{m_{P}}$ be the index variable to denote the instances of $m_{m_{P}}$. Let $q_{m_{P}}^{L}$ and $q_{m_{P}}^{H}$ denote the lowest- and highest-numbered instances of $m_{m_{P}}$ respectively. Let $q_{m_{S}}, q_{m_{S}}^{L}$ and $q_{m_{S}}^{H}$ denote the index variable for instances, and lowest- and highest-numbered instances of $m_{m_{S}}$ respectively. The calculations for $R_{m_{P}}$ and $R_{m_{S}}$ are adapted from the periodic and sporadic cases respectively as follows.

$$
\begin{aligned}
& R_{m_{P}}=\max \left\{R_{m_{P}}\left(q_{m_{P}}\right)\right\}, \forall q_{m_{P}}^{L} \leq q_{m_{P}} \leq q_{m_{P}}^{H} \\
& R_{m_{S}}=\max \left\{R_{m_{S}}\left(q_{m_{S}}\right)\right\}, \forall q_{m_{S}}^{L} \leq q_{m_{S}} \leq q_{m_{S}}^{H}
\end{aligned}
$$

The calculations for the worst-case response time of each instance of $m_{m_{P}}$ and $m_{m_{S}}$ are adapted from (2) and (13) as follows.

$$
R_{m_{P}}\left(q_{m_{P}}\right)=S T_{m_{P}}+C_{m}-\left(\varphi_{m_{P}}\left(\phi_{i}\right)+\left(q_{m_{P}}-1\right) \cdot T_{m}\right)
$$

$$
R_{m_{S}}\left(q_{m_{S}}\right)=S T_{m_{S}}+C_{m}-\left(q_{m_{S}}-1\right) \cdot M U T_{m}
$$

Where $\varphi_{m_{P}}\left(\phi_{i}\right)$ is calculated using (3). The calculations for $S T_{m_{P}}$ and $S T_{m_{S}}$ are adapted from (4) after some augmentation.

$$
\begin{array}{r}
S T_{m_{P}}^{n+1}=B_{m}+Q_{m_{S}}^{P} \cdot C_{m}+\left(q_{m_{P}}-q_{m_{P}}^{L}\right) \cdot C_{m}+ \\
\sum_{\forall \Gamma_{k} \in \Gamma} W_{m_{P}}\left(\Gamma_{k}, \phi_{k}, S T_{m_{P}}^{n}\right) \\
S T_{m_{S}}^{n+1}=B_{m}+Q_{m_{P}}^{S} \cdot C_{m}+\left(q_{m_{S}}-q_{m_{S}}^{L}\right) \cdot C_{m}+ \\
\sum_{\forall \Gamma_{k} \in \Gamma} W_{m_{S}}\left(\Gamma_{k}, \phi_{k}, S T_{m_{S}}^{n}\right)
\end{array}
$$

\section{Effect of Self Interference in a Mixed Message}

$Q_{m_{S}}^{P} \cdot C_{m}$ and $Q_{m_{P}}^{S} \cdot C_{m}$ in (22) and (23) respectively represent the effect of self interference in a mixed message. By self interference we mean that the periodic copy of a mixed message can be interfered by the sporadic copy and vice versa. Since, both $m_{m_{P}}$ and $m_{m_{S}}$ have equal priorities, any instance of $m_{m_{S}}$ queued ahead of $m_{m_{P}}$ will contribute an extra delay to the worst-case queueing delay experienced by $m_{m_{P}}$. A similar argument holds in the case of $m_{m_{S}}$. We reuse the effect of self interference in a mixed message that we derived in [20] as follows.

In order to derive the contribution of one copy of a mixed message to the worst-case queueing delay of the other, consider three different cases, depicting the transmission pattern of a mixed message $m$, shown in Figure 4. In the first case, we assume $T_{m}$ to be greater than $M U T_{m}$. That is, there can be more transmissions of $m_{m_{S}}$ compared to that of $m_{m_{P}}$. Since, the maximum update time between the queueing of any two instances of $m_{m_{S}}$ can be arbitrarily very long, it is possible to have fewer sporadic transmissions than periodic transmissions of $m$. In the second case, we assume that $T_{m}$ is equal to $M U T_{m}$. In this case, there are equal number of transmissions of $m_{m_{P}}$ and $m_{m_{S}}$. In 
the third case, we assume that $T_{m}$ is smaller than $M U T_{m}$. This implies that the number of sporadic transmissions will be less than the periodic transmissions of $m$.

It is important to note that in the example shown in Figure 4 , there is a small offset between the first periodic and sporadic transmission of $m$. This offset is used to maximize the queueing delay. If this offset is removed then only one message will be queued corresponding to the first instance of both $m_{m_{P}}$ and $m_{m_{S}}$. Moreover, the larger value between $T_{m}$ and $M U T_{m}$ is the integer multiple of the smaller in all the cases. This relationship along with the offset between $T_{m}$ and $M U T_{m}$ ensures that periodic and sporadic transmissions of $m$ will not overlap, there by, maximizing the queueing delay.

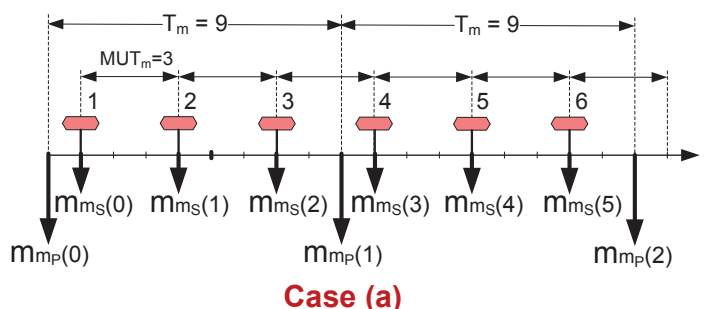

Case (a)

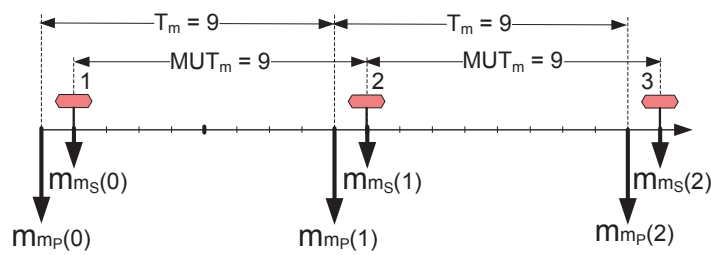

Case (b)

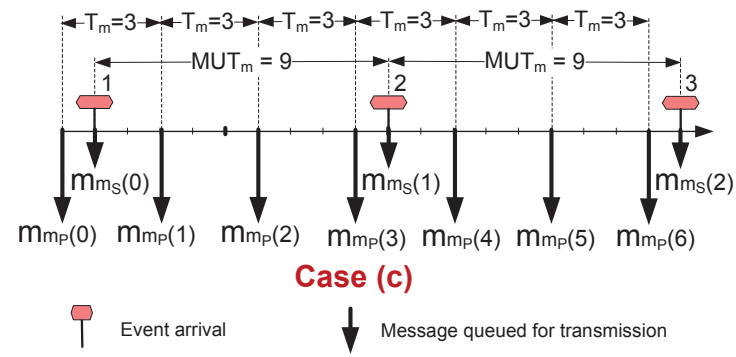

Figure 4. Self interference in a mixed message: (a) $\mathbf{T}_{\mathrm{m}}>\mathrm{MUT}_{\mathrm{m}}$, (b) $\mathrm{T}_{\mathrm{m}}=\mathrm{MUT}_{\mathrm{m}}$, (c) $\mathbf{T}_{\mathbf{m}}<\mathbf{M U T}_{\mathbf{m}}$

\section{Case (a): $\mathbf{T}_{\mathbf{m}}>\mathrm{MUT}_{\mathbf{m}}$}

Let the message under analysis be $m_{m_{P}}$ and consider case (a) in Figure 4. An application task queues $m$ periodically with a period $T_{m}$ (equal to 9 time units). Moreover, the same task can also queue $m$ sporadically at the arrival of events (labeled with numbers 1-6). The queueing of $m_{m_{E}}$ is constrained by $M U T_{m}$ (equal to 3 time units). The first instance of $m_{m_{P}}\left(q_{m_{P}}=0\right)$ is queued for transmission as shown by $m_{m_{P}}(0)$ in Figure 4 . If event 1 had arrived at the same time as the queueing of $m_{m_{P}}(0)$ then the signals in $m_{m_{S}}(0)$ would have been updated as part of $m_{m_{P}}(0)$. In that case, $m_{m_{S}}(0)$ would not have been queued separately (this is the property of the mixed message in the HCAN protocol). In order to maximize the contribution of $m_{m_{S}}$ on the queueing delay of $m_{m_{P}}, m_{m_{S}}(0)$ is queued just after the queueing of $m_{m_{P}}(0)$ as shown in all the cases in Figure 4. Therefore, $m_{m_{S}}(0)$ and subsequent instances of $m_{m_{S}}$ will have no contribution in the worst-case queueing delay of the first instance of $m_{m_{P}}$ denoted by $m_{m_{P}}(0)$.

Consider the second instance of $m_{m_{P}}$. All the instances of $m_{m_{S}}$ that are queued ahead of $m_{m_{P}}$ (1) will contribute to its worst-case queueing delay. It can be observed in the case (a) that the first three instances of $m_{m_{S}}$ are queued ahead of $m_{m_{P}}(1)$. Similarly, there are six instances of $m_{m_{S}}$ that are queued ahead of $m_{m_{P}}(2)$.

Let $Q_{m_{S}}^{P}$ denotes the total number of instances of $m_{m_{S}}$ that are queued ahead of the $q_{m_{P}}^{t h}$ instance of $m_{m_{P}}$. We can generalize $Q_{m_{S}}^{P}$ for the case (a) as follows.

$$
Q_{m_{S}}^{P}=\left\lceil\frac{q_{m_{P}} T_{m}}{M U T_{m}}\right\rceil
$$

For example, consider again the queueing of different instances of $m_{m_{S}}$ and $m_{m_{P}}$ in the case (a). Equation (24) yields the set $\left\{Q_{m_{S}}^{P}=0,3,6, \ldots\right\}$ for the corresponding values in the set $\left\{q_{m_{P}}=0,1,2, \ldots\right\}$. Thus the total number of instances of $m_{m_{S}}$ queued ahead of each instance of $m_{m_{p}}$ calculated by (24) are consistent with the case (a) in Figure 4.

Case (b): $\mathbf{T}_{\mathbf{m}}=\mathrm{MUT}_{\mathbf{m}}$

Consider case (b) in which $T_{m}$ is equal to $M U T_{m}$. It can be observed from Figure 4 that there are 0,1 , and 2 instances of $m_{m_{S}}$ that are queued ahead of $m_{m_{P}}(0)$, $m_{m_{P}}(1)$ and $m_{m_{P}}(2)$ respectively. When Equation (24) is used in case (b), we get the set $\left\{Q_{m_{S}}^{P}=0,1,2, \ldots\right\}$ for the corresponding values in the set $\left\{q_{m_{P}}=0,1,2, \ldots\right\}$. Therefore, (24) is also applicable on case (b).

Case (c): $\mathbf{T}_{\mathbf{m}}<$ MUT $_{\mathbf{m}}$

Now, consider case (c) in which $T_{m}$ (3 time units) is smaller than $M_{U} T_{m}$ (9 time units). The first instance of $m_{m_{S}}$ denoted by $m_{m_{S}}(0)$ will be queued ahead of $m_{m_{P}}(1), m_{m_{P}}(2)$ and $m_{m_{P}}(3)$. Similarly, the two instances of $m_{m_{S}}$ denoted by $m_{m_{S}}(0)$ and $m_{m_{S}}(1)$ will contribute to the worst-case queueing delay of $m_{m_{P}}(4), m_{m_{P}}(5)$ and $m_{m_{P}}(6)$. (24) yields the set $\left\{Q_{m_{S}}^{P}=0,1,1,1,2,2,2, \ldots\right\}$ for the corresponding values in the set $\left\{q_{m_{P}}=0,1,2,3,4,5,6, \ldots\right\}$. Thus the total number of instances of $m_{m_{S}}$ queued ahead of each instance of $m_{m_{P}}$ calculated by equation (24) are consistent with the case (c) in Figure 4.

Now we consider the effect of jitter on the instances of $m_{m_{S}}$ prior to $m_{m_{S}}(0)$ which can be queued just ahead of $m_{m_{P}}(0)$ and contribute to the worst-case queueing delay of $m_{m_{P}}$. We assume FIFO queueing policy among the instances of the same message. Due to the offset of $m_{m_{P}}$, there may be one or more instances of $m_{m_{S}}$ that can be queued ahead of the first instance of $m_{m_{P}}$. Hence, the offset of $m_{m_{P}}$ should also be taken into account when considering the self interference from $m_{m_{S}}$. By considering the jitter of $m_{m_{S}}$ and offset of $m_{m_{P}}$ to $Q_{m_{S}}^{P}$, equation (24) 
can be generalized for the three cases as follows.

$$
Q_{m_{S}}^{P}=\left\lceil\frac{q_{m_{P}} T_{m}+J_{m}+O_{m}}{M U T_{m}}\right\rceil
$$

The total number of instances of $m_{m_{P}}$ that are queued ahead of the $q_{m_{S}}^{t h}$ instance of $m_{m_{S}}$, denoted by $Q_{m_{P}}^{S}$, can be derived in a similar fashion. However, $Q_{m_{P}}^{S}$ does not contain the term $O_{m}$ because sporadic messages do not have any offset relations with any other message. Thus $Q_{m_{P}}^{S}$ can be calculated by the following equation.

$$
Q_{m_{P}}^{S}=\left\lceil\frac{q_{m_{S}} M U T_{m}+J_{m}}{T_{m}}\right\rceil
$$

Using the values of $Q_{m_{P}}^{S}$ and $Q_{m_{S}}^{P}$ from (26) and (25) in (22) and (23) respectively as follows.

$$
\begin{aligned}
& S T_{m_{P}}^{n+1}=B_{m}+\left\lceil\frac{q_{m_{P}} \cdot T_{m}+J_{m}+O_{m}}{M U T_{m}}\right\rceil \cdot C_{m} \\
& +\left(q_{m_{P}}-q_{m_{P}}^{L}\right) \cdot C_{m}+\sum_{\forall \Gamma_{k} \in \Gamma} W_{m_{P}}\left(\Gamma_{k}, \phi_{k}, S T_{m_{P}}^{n}\right) \\
& S T_{m_{S}}^{n+1}=B_{m}+\left\lceil\frac{q_{m_{S}} \cdot M U T_{m}+J_{m}}{T_{m}}\right\rceil \cdot C_{m} \\
& +\left(q_{m_{S}}-q_{m_{S}}^{L}\right) \cdot C_{m}+\sum_{\forall \Gamma_{k} \in \Gamma} W_{m_{S}}\left(\Gamma_{k}, \phi_{k}, S T_{m_{S}}^{n}\right)
\end{aligned}
$$

The calculations for $W_{m_{P}}, q_{m_{P}}^{L}, q_{m_{P}}^{H}$ and $L_{m_{P}}$ are done using (6), (8), (9) and (10) by replacing the index $m$ with $m_{P}$ respectively. Similarly, $W_{m_{S}}, q_{m_{S}}^{L}, q_{m_{S}}^{H}$ and $L_{m_{S}}$ are calculated using (6), (14), (15) and (16) by replacing the index $m$ with $m_{S}$ respectively. Further, the calculations in (5), (7) and (11) hold intact with proper replacement of the index variable for both $m_{m_{P}}$ and $m_{m_{S}}$.

\section{Automotive-application case study}

In this section, we conduct the Steer-By-Wire (SBW) case study. The SBW system is an automotive feature that substitutes most of the mechanical and hydraulic components with the electronic components in the steering system of the vehicle. We adapt the SBW system from [10]. The partial architecture of the SBW system is shown in Figure 5. There are two ECUs (rest of the ECUs are not shown for simplicity) that are connected to the CAN network.

The Steering Control ECU denoted by $E C U_{S}$ receives input from three sensors that correspond to the steering angle, steering torque (applied by the driver) and vehicle speed signals. It sends three messages $m_{1}, m_{2}$ and $m_{3}$ to the network. These messages carry information regarding steering angle, torque and feedback signals. $m_{1}$ is a periodic, $m_{2}$ is a mixed and $m_{3}$ is a sporadic message. $E C U_{S}$ receives the periodic message $m_{4}$ that contains information about wheel torque that is sent by the Wheel Control ECU. Based on these inputs, it calculates the feedback steering torque and sends it to the feedback actuator. The feedback torque actuator is responsible for producing the turning effect of the steering which in turn produces the feeling of turning the wheels for the driver.

Similarly, the Wheel Control ECU denoted by $E C U_{W}$ acquires signals from wheel angle and wheel torque sensors. Depending upon these signals and the signals received in the CAN message, it calculates the wheel torque, and produces actuation signals for the wheel control actuators. Apart from $m_{4}$, it also sends a periodic message $m_{5}$ to the network. The timing attributes of all messages are shown in Table 1. We analyzed this message set with the extended analysis. The response times calculated using the extended analysis are also listed in Table 1 . Since, the jitter of messages $m_{2}$ and $m_{5}$ is higher than the corresponding periods, there are several instances of these messages present in the corresponding busy periods. For example, there are four instances of $m_{2}$ that are present in the priority level-2 busy period. The extended analysis calculates the response times of all these instances and considers maximum among them as the worst-case response time of $m_{2}$. It should be noted that correct analysis of this message set would not have been possible with the existing analysis because it contains a mixed message whose jitter and deadline are greater than the corresponding period.

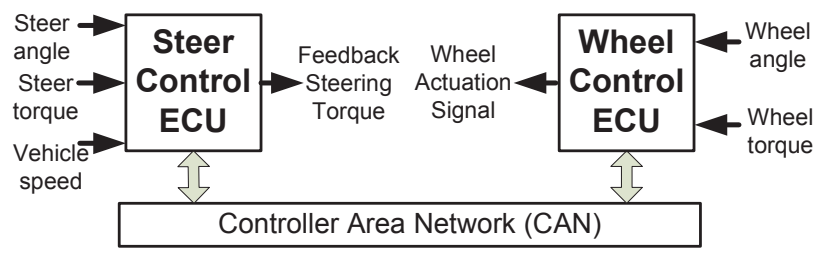

Figure 5. Partial architecture of the steer-by-wire system

\section{Conclusion}

The existing response-time analysis of CAN does not support the analysis of mixed messages that are scheduled with offsets and have jitter and deadlines higher than their transmission periods. Message jitter can be higher than its period in practical systems. We extended the existing offset-based analysis for CAN by lifting the restrictions on message jitter and deadline. The extended analysis provides safe upper bounds on the response times of mixed messages that are scheduled with offsets. Mixed messages are implemented by several higher-level protocols for CAN that are used in the automotive industry today. The extended analysis is applicable to any higher-level protocol for CAN that uses periodic, sporadic, and mixed transmission of messages that are scheduled with offsets. We also conducted the automotive-application case study to demonstrate the applicability of our analysis.

In the future, we plan to develop an optimized offset assignment method for the systems that contain periodic as well as mixed messages. We also plan to implement the extended analysis as a plug-in for the existing industrial tool suite the Rubus-ICE [5]. 


\begin{tabular}{|c|c|c|c|c|c|c|c|c|c||c|}
\hline $\mathrm{ECU}$ & $m$ & $P_{m}$ & $\xi_{m}$ & $T_{m}$ & $M U T_{m}$ & $C_{m}$ & $O_{m}$ & $J_{m}$ & $D_{m}$ & $R_{m}$ \\
\hline$E C U_{S}$ & $m_{1}$ & 1 & $P$ & 10 & - & 1 & 0 & 1 & 10 & 3 \\
\hline$E C U_{S}$ & $m_{2}$ & 2 & $M$ & 10 & 10 & 1 & 1 & 11 & 20 & 15 \\
\hline$E C U_{S}$ & $m_{3}$ & 3 & $S$ & - & 10 & 1 & 0 & 0 & 10 & 7 \\
\hline$E C U_{W}$ & $m_{4}$ & 4 & $P$ & 10 & - & 1 & 0 & 0 & 10 & 8 \\
\hline$E C U_{W}$ & $m_{5}$ & 5 & $P$ & 10 & - & 1 & 2 & 12 & 20 & 18 \\
\hline
\end{tabular}

Table 1. Attributes and response times of periodic, sporadic and mixed messages in the steer-by-wire system

\section{Acknowledgement}

The work in this paper is supported by the Swedish Knowledge Foundation (KKS) within the projects SythSoft and FEMMVA. The authors would like to thank the industrial partners Arcticus Systems AB, BAE Systems Hägglunds and Volvo Construction Equipment (VCE), Sweden.

\section{References}

[1] Automotive networks. CAN in Automation (CiA). http://www.cancia.org/index.php?id=416.

[2] AUTOSAR Techincal Overview, Version 2.2.2., Release 3.1, The AUTOSAR Consortium, Aug., 2008. http://autosar.org.

[3] CANopen Application Layer and Communication Profile. CiA Draft Standard 301. Version 4.02. February 13, 2002. http://www.can-cia.org/index.php?id=440.

[4] Requirements on Communication, Release 3.0, Rey 7, Ver. 2.2.0. The AUTOSAR Consortium, Sep., 2010. www.autosar.org/download/R3.0/AUTOSAR_SRS_COM.pdf.

[5] Rubus-ICE. http://www.arcticus-systems.com.

[6] Volcano Network Architect (VNA). Mentor Graphics. http://www. mentor.com/products/vnd/communication-management/vna.

[7] Hägglunds Controller Area Network (HCAN), Network Implementation Specification. BAE Systems Hägglunds, Sweden (internal document), April 2009.

[8] N. Audsley, A. Burns, R. Davis, K. Tindell, and A. Wellings. Fixed priority pre-emptive scheduling:an historic perspective. Real-Time Systems, 8(2/3):173-198, 1995.

[9] N. Audsley, A. Burns, M. Richardson, K. Tindell, and A. J. Wellings. Applying new scheduling theory to static priority preemptive scheduling. Software Engineering Journal, 8(5):284-292, 1993.

[10] K. Chaaban, P. Leserf, and S. Saudrais. Steer-by-wire system development using AUTOSAR methodology. In 14th IEEE Conference on Emerging Technologies and Factory Automation, 2009.

[11] Y. Chen, R. Kurachi, H. Takada, and G. Zeng. Schedulability comparison for can message with offset: Priority queue versus fifo queue. In 19th International Conference on Real-Time and Network Systems (RTNS), pages 181-192, Sep. 2011.

[12] R. Davis, A. Burns, R. Bril, and J. Lukkien. Controller Area Network (CAN) schedulability analysis: Refuted, revisited and revised. Real-Time Systems, 35:239-272, 2007.

[13] R. I. Davis, S. Kollmann, V. Pollex, and F. Slomka. Controller Area Network (CAN) Schedulability Analysis with FIFO queues. In 23rd Euromicro Conference on Real-Time Systems, July 2011.

[14] L. Du and G. Xu. Worst case response time analysis for can messages with offsets. In IEEE International Conference on Vehicular Electronics and Safety (ICVES), pages $41-45$, nov. 2009.

[15] M. Grenier, L. Havet, and N. Navet. Pushing the limits of can- scheduling frames with offsets provides a major performance boost. In 4th European Congress on Embedded Real Time Software (ERTS), 2008.
[16] M. Grenier, L. Havet, and N. Navet. Automotive embedded systems handbook. In N. Navet and F. Siminot-Lion, editors, Chapter 14: Scheduling messages with offsets on Controller Area Network - a major performance boost. CRC Press, 2009.

[17] R. Henia, A. Hamann, M. Jersak, R. Racu, K. Richter, and R. Ernst. System level performance analysis - the symta/s approach. Computers and Digital Techniques, 152(2):148-166, March 2005.

[18] ISO 11898-1. Road Vehicles interchange of digital information controller area network (CAN) for high-speed communication, ISO Standard-11898, Nov. 1993.

[19] M. Joseph and P. Pandya. Finding response times in a real-time system. The Computer Journal, 29(5):390-395, 1986.

20] S. Mubeen, J. Mäki-Turja, and M. Sjödin. Extending schedulability analysis of controller area network (CAN) for mixed (periodic/sporadic) messages. In 16th IEEE Conference on Emerging Technologies and Factory Automation (ETFA), sept. 2011.

[21] S. Mubeen, J. Mäki-Turja, and M. Sjödin. Support for Holistic Response-time Analysis in an Industrial Tool Suite: Implementation Issues, Experiences and a Case Study. In 19th IEEE Conference on Engineering of Computer Based Systems (ECBS), pages 210 -221, April 2012.

[22] S. Mubeen, J. Mäki-Turja, and M. Sjödin. Worst-case responsetime analysis for mixed messages with offsets in controller area network. In 17th IEEE Conference on Emerging Technologies and Factory Automation (ETFA), sept. 2012.

[23] S. Mubeen, J. Mäki-Turja, and M. Sjödin. Support for end-to-end response-time and delay analysis in the industrial tool suite: Issues, experiences and a case study. Computer Science and Information Systems, ISSN: 1361-1384, 10(1), 2013.

[24] M. Nolin, J. Mäki-Turja, and K. Hänninen. Achieving Industrial Strength Timing Predictions of Embedded System Behavior. In ESA, pages 173-178, 2008.

[25] J. Palencia and M. G. Harbour. Schedulability Analysis for Tasks with Static and Dynamic Offsets. IEEE International Symposium on Real-Time Systems (RTSS), 1998.

[26] Robert Bosch GmbH. CAN Specification Version 2.0. Postfach 30 02 40, D-70442 Stuttgart, 1991.

[27] S. Mubeen, J. Mäki-Turja and M. Sjödin. Response-Time Analysis of Mixed Messages in Controller Area Network with Priorityand FIFO-Queued Nodes. In 9th IEEE International Workshop on Factory Communication Systems (WFCS), May 2012.

[28] L. Sha, T. Abdelzaher, K.-E. A. rzén, A. Cervin, T. P. Baker, A. Burns, G. Buttazzo, M. Caccamo, J. P. Lehoczky, and A. K. Mok. Real Time Scheduling Theory: A Historical Perspective. Real-Time Systems, 28(2/3):101-155, 2004.

[29] A. Szakaly. Response Time Analysis with Offsets for CAN. Master's thesis, Department of Computer Engineering, Chalmers University of Technology, Nov. 2003.

[30] K. Tindell and A. Burns. Guaranteeing Message Latencies on Controller Area Network (CAN). In 1st International CAN Conference, 1994, pages $1-11$.

[31] K. Tindell, H. Hansson, and A. Wellings. Analysing real-time communications: controller area network (CAN). In Real-Time Systems Symposium (RTSS) 1994, pages 259-263.

[32] P. Yomsi, D. Bertrand, N. Navet, and R. Davis. Controller Area Network (CAN): Response Time Analysis with Offsets. In 9th IEEE International Workshop on Factory Communication Systems (WFCS), May 2012. 